\title{
A Homogeneous Split-Luciferase Assay for Rapid and Sensitive Detection of Anti-SARS CoV-2 Antibodies
}

Igor Stagljar ( $\sim$ stagljar.igor@utoronto.ca )

University of Toronto

Shawn Owen ( $\nabla$ shawn.owen@utah.edu )

University of Utah

Zhong Yao

University of Toronto

Luka Drecun

University of Toronto

Farzaneh Aboualizadeh

University of Toronto

Sun Jin Kim

University of Utah

Zhijie Li

University of Toronto

Emelissa J Valcourt

National Microbiology Laboratory

Kathy Manguiat

National Microbiology Laboratory

Heidi Wood

National Microbiology Laboratory

Simon Plenderleith

Sunnybrook Research Institute

Lily Yip

Sunnybrook Research Institute

Xinliu Li

Mount Sinai Hospital

Zoe Zhong

Mount Sinai Hospital

Feng Yun Yue

University of Toronto

Jamie Snider 
University of Toronto

Jelena Tomic

Univeristy of Toronto

Michael A. Drebot

National Microbiology Laboratory

\section{Allison McGeer}

Mount Sinai Hospital

Mario Ostrowski

University of Toronto

\section{Samira Mubareka}

Sunnybrook Research Institute

James Rini

University of Toronto

\section{Method Article}

Keywords: SARS-CoV-2, antibody, IgG, NanoLuciferase

Posted Date: December 1st, 2020

DOI: https://doi.org/10.21203/rs.3.rs-115221/v1

License: (1) (i) This work is licensed under a Creative Commons Attribution 4.0 International License. Read Full License

Version of Record: A version of this preprint was published at Nature Communications on December 1st, 2020. See the published version at https://doi.org/10.1038/s41467-021-22102-6. 


\section{A Homogeneous Split-Luciferase Assay for Rapid and Sensitive Detection of Anti- SARS CoV-2 Antibodies}

Zhong Yao ${ }^{1,2}$, Luka Drecun ${ }^{1,3}$, Farzaneh Aboualizadeh ${ }^{1,2}$, Sun Jin Kim ${ }^{4}$, Zhijie Li ${ }^{3}$, Heidi Wood $^{5}$, Emelissa J. Valcourt ${ }^{5}$, Kathy Manguiat ${ }^{5}$, Simon Plenderleith ${ }^{6}$, Lily Yip ${ }^{6}$, Xinliu $\mathrm{Li}^{7}$, Zoe Zhong ${ }^{7}$, Feng Yun Yue ${ }^{8}$, Jamie Snider ${ }^{1,2}$, Jelena Tomic ${ }^{1,2}$, Michael A. Drebot ${ }^{5}$, Allison McGeer ${ }^{7}$, Mario Ostrowski ${ }^{8}$, Samira Mubareka ${ }^{6,8}$, James Rini',3, Shawn Owen $^{4,9 *}$, Igor Stagljar ${ }^{1,2,3,10 *}$

${ }^{1}$ Donnelly Centre, University of Toronto, Toronto, ON, Canada

${ }^{2}$ Department of Biochemistry, University of Toronto, Toronto, ON, Canada ${ }^{3}$ Department of Molecular Genetics, University of Toronto, Toronto, ON, Canada ${ }^{4}$ Department of Pharmaceutics and Pharmaceutical Chemistry, University of Utah, USA ${ }^{5}$ Zoonotic Diseases and Special Pathogens division, National Microbiology Laboratory, Public Health Agency of Canada, Winnipeg, MB, Canada ${ }^{6}$ Biological Sciences, Sunnybrook Research Institute, Toronto, ON, Canada ${ }^{7}$ Department of Microbiology, Mount Sinai Hospital, Toronto, ON, Canada ${ }^{8}$ Department of Laboratory Medicine and Pathobiology, University of Toronto, Toronto, ON, Canada

${ }^{9}$ Department of Biomedical Engineering, Department of Medicinal Chemistry, University of Utah, Salt Lake City, UT, USA

${ }^{10}$ Mediterranean Institute for Life Sciences, Meštrovićevo Šetalište 45, HR-21000 Split, Croatia

${ }^{*}$ corresponding authors:

Shawn Owen

Phone: 1-801-581-8069

E-mail: shawn.owen@hsc.utah.edu

Igor Stagljar

Phone: 1-416-946-7828

Fax: 1-416-978-8287

E-mail: igor.stagliar@utoronto.ca 


\begin{abstract}
To meet the urgent demand for better diagnostic tools to combat the ongoing COVID-19 pandemic, we developed a homogeneous immunoassay to detect IgG antibodies against SARS-CoV-2. This assay is based on a tri-part Nanoluciferase (tNLuc) approach, in which the spike protein of SARS-CoV-2 and protein G, fused respectively to two different tNLuc tags, are used as antibody probes. Target engagement of the probes allows reconstitution of a functional luciferase in the presence of the third tNLuc component. The assay is performed directly in liquid phase of patient sera and enables rapid, quantitative and low-cost detection. We show that tNLuc maintains a similar sensitivity to ELISA, while its readouts are highly consistent with various neutralizing antibody assays. This proof-of-principle study suggests potential applications in diagnostics and disease and vaccination management.
\end{abstract}


Testing is crucial for combating the coronavirus disease 2019 (COVID-19) pandemic ${ }^{1}$. Serological tests are used to determine the level of antibodies against severe acute respiratory syndrome coronavirus 2 (SARS-CoV-2) in blood ${ }^{2}$. Their results reflect the disease progress or its history, as well as the immunity of a patient ${ }^{3}$, which is valuable information for diagnosis and disease management. Importantly, with the advent of different upcoming vaccines, serological testing is becoming a necessary tool for evaluating acquired immunity at both individual and population levels. In addition, serological testing is essential for epidemic studies and related policymaking.

Numerous serological assays have been developed ${ }^{4}$. Among them, lateral flow immunoassays (LFIAs) are rapid and easy to perform, and therefore have found use as point-of-care tests ${ }^{5}$. However, their lack of quantifiability, coupled with their relatively low sensitivity and specificity, limits their usage as standard and reliable tests to evaluate antibody titers ${ }^{6,7}$. Alternatively, enzyme-linked immunosorbent assays (ELISAs) are standard quantitative serological methods displaying good sensitivity and specificity ${ }^{8,9}$. They too, however, have notable shortcomings including long processing time (3-5 hours), tedious procedures (multiple wash-aspirate cycles) and extra steps involved in pre-processing of binding plates. Several chemiluminescent immunoassay (CLIA) platforms targeting COVID-19 have also been developed by companies such as Abbott ${ }^{10}$, DiaSorin ${ }^{11}$, Roche $^{12}$ and Siemens ${ }^{13}$. These are highly automated assays suitable for measurement of large content samples and are characterized by good quantifiability and sensitivity ${ }^{13}$. However, the measurements require highly specialized and expensive instruments, which limit their widespread application.

Here, we describe a liquid-phase serological assay for lgG antibodies against SARS-CoV-2 based on split tripart Nanoluciferase (tNLuc) that can be performed directly with patient sera. We demonstrate that the assay displays quantifiability and sensitivity comparable to ELISA, is rapid/easy to perform, is very cost-efficient and produces readouts highly consistent with neutralizing antibody tests, strongly supporting its potential value in COVID-19 diagnosis and disease and vaccination management.

\section{Results}

Protein complementation assays (PCAs) are a strategy to detect protein-protein interactions (PPIs) ${ }^{14,15}$. In this approach, a 'sensor' protein is split into two fragments, which are then fused to two candidate interacting proteins of interest. The binding of the two proteins of interest arranges the sensor fragments in a favourable position that allows them to reconstitute into a functional protein which can produce a detectable 
signal representative of the $\mathrm{PPI}^{16}$. Different sensors such as fluorescent proteins, transcription factors, proteases and more have been successfully used in various designs. Among them, split luciferases have been shown to have the advantages of high signal/noise ratio and rapid reconstitution, making them widely used ${ }^{17-19}$. However, the conventional strategy of splitting luciferase into two fragments has limitations. For instance, the relatively large size of the fragments may interfere with target protein folding or function and/or the interaction with partner molecules. The residual intrinsic affinity between the two luciferase fragments may also lead to increased background signal. A recently developed tri-part strategy circumvents these limitations by splitting NanoLuc ${ }^{\circledR}$ (NLuc), the brightest luciferase identified so far, into three fragments: two short peptides ( $\beta 9$ and $\beta 10$ each containing 11 amino acids) and one $16 \mathrm{kDa}$ fragment $(\Delta 11 S)^{20,21}$. Here we report the adaptation of this variant tri-part NanoLuc ${ }^{\circledR}$ (tNLuc) for use in our COVID-19 antibody detection system (Fig. 1A). In our design, the $\beta 9$ and $\beta 10$ tags are separately fused to a pair of probes which can respectively recognize an IgG molecule against SARS-CoV-2 at different sites. The first probe is generated by fusing the $\beta 9$ tag to the $C 2$ domain of protein $G$, which exclusively binds to all the isotypes of human $\lg G$ but not to $\lg M, \lg A$ or $\lg E$ immunoglobulins ${ }^{22}$. The second probe is specific to antibodies that bind the SARS-CoV-2 spike (S) protein, the viral membrane protein responsible for host cell receptor binding ${ }^{23}$ and which is also the target of most of the neutralizing antibodies found in patients ${ }^{24}$. We generated two forms of this probe by fusing the $\beta 10$ tag to either the ectodomain of the $S$ protein or to its receptor binding domain (RBD). The assay itself is remarkably straightforward to perform and involves only two simple steps (Fig. 1A): (i) diluted serum or plasma samples are mixed with the two probes and incubated for 30 minutes; (ii) an aliquot of this mixture is combined with the third component, $\Delta 11 \mathrm{~S}$, and the luciferase substrate and incubated for another 30 minutes, followed by luminescence recording with a luminometer. The whole process occurs directly in the liquid phase and does not require any washing steps.

As the peptide tags ( $\beta 9$ and $\beta 10$ ) can be fused either at the $\mathrm{N}$ - or $\mathrm{C}$-terminus of the probes or at both positions (Supplementary Fig. 1), we first sought to determine which combination of the various probes would result in the most signal with the least background. We tested all combinations of the probes with CR3022, an antibody that binds to the RBD of the SARS-CoV-2 S protein ${ }^{25}$, and observed that all combinations produced signal, albeit to varying extents (Fig. 1B). This suggests that despite the fact that the probes bind to different domains of $\mathrm{gGG}$, the molecules are sufficiently flexible to allow proximal localization of the two tags, enabling reconstitution of tNLuc into an 
active luciferase. Of all the combinations, two produced the highest signal-tobackground: $\beta 9-G$ with $\beta 10-S$ and $\beta 9-G$ with $\beta 10-S-\beta 10$. Further testing of these two combinations using different concentrations of CR3022 demonstrated typical dose response curves (Fig. 1C, D). However, based on the calculated Kd values using $\beta 10-S$ $(0.42 \mu \mathrm{g} / \mathrm{mL})$ and $\beta 10-S-\beta 10(0.23 \mu \mathrm{g} / \mathrm{mL})$, we predicted that $\beta 10-S-\beta 10$ would have greater potential to detect antibody at lower concentrations (Fig. 1E). Thus, the $\beta 9$ $\mathrm{G} / \beta 10-\mathrm{S}-\beta 10$ probe pair was chosen to be used in the final assay.

Human plasma contains a high concentration of $\mathrm{lgG}(4-22 \mathrm{mg} / \mathrm{mL}$, median 11 $\mathrm{mg} / \mathrm{mL}$ in serum $)^{26,27}$, the vast majority of which will not be specific for the SARS-CoV-2 S-protein. Since they can, however, bind the protein G probe, these "non-specific" antibodies will reduce the sensitivity of our assay. We investigated the effect of $\lg G$ interference on the assay by adding different amounts of human IgG into the reaction mixture and as predicted inhibition was indeed observed (Fig. 2A). The detailed kinetics of inhibition were further analyzed based on the dose response testing of CR3022 in the presence of different amounts of IgG (Fig. 2B). We performed mathematical analysis by fitting different inhibition models to these experimental data. A model of allosteric noncompetitive inhibition provided the best fit, as judged by visual comparison of the curve positions/shapes with the plotted data points (Fig. 2B) and the calculated $\mathrm{R}^{2}$ (0.9911). The allosteric effect might be derived from the binding of protein $G$ to two sites on an IgG molecule while the detailed molecular mechanism of noncompetitive inhibition merits further exploration.

Binding parameters derived from the model $(\mathrm{Ki}=58.5 \mu \mathrm{g} / \mathrm{mL}$, and $\mathrm{Kd}=0.22 \mu \mathrm{g} / \mathrm{mL}$ which is consistent with the results in Fig. 1E) suggest a considerable difference of the probe binding affinities toward CR3022 antibody and general IgG. We therefore reasoned that the inhibition can be eliminated or alleviated simply by dilution, a step also required to reduce nonspecific signal when performing ELISA on blood samples. This was supported by computational simulation of assays, using the obtained mathematical model, on virtual samples containing different concentrations of $\lg G$ and varying amounts of CR3022 (Supplementary Fig. 2A). The recovery rates (the luminescence ratio of a sample and the correspondent control without additional $\lg G$ ) at different dilution endpoints were calculated (Supplementary Fig. 2B). At 1:100 dilution, the recovery rates for the samples of $20 \mathrm{mg} / \mathrm{mL} \operatorname{lgG}$ (close to the upper limit in human serum) are around $20 \%$. This was significantly improved by further dilution, reaching $47-$ $60 \%$ at $1: 300$ dilution and $86-88 \%$ at $1: 900$ dilution. Reduced IgG brought even better recovery; samples containing $10 \mathrm{mg} / \mathrm{mL} \operatorname{lgG}$ (close to the median IgG in human serum) 
showed $\sim 80 \%$ recovery at $1: 300$ dilution and $5 \mathrm{mg} / \mathrm{mL}$ of $\operatorname{lgG}$ (close to the lower limit of IgG in human serum) showed more than $90 \%$ recovery at 1:300 dilution.

Building on these results, we then adopted a strategy involving sample dilution to obtain more accurate measurements of the $\mathrm{S}$ protein specific antibodies. The overall tNLuc signal of a sample was calculated using the signal summation algorithm (luminescence sum of RLU values at 1:300, 1:900 and 1:2700 dilutions) to avoid parameter estimation, as required by other algorithms, while still maintaining a power similar to curve fitting ${ }^{28}$. The signal at the lowest dilution endpoint (1:100) was excluded from the analysis to avoid the substantial signal interference caused by $\lg G$ under these conditions. Computational simulation (Supplementary Fig. 2C) demonstrated good recovery; samples with medium and low IgG levels showed more than $80 \%$ recovery while samples with high IgG levels showed about $60 \%$ (for low CR3022 dose) to $70 \%$ (for high CR3022 dose) recovery. Performance was further evaluated experimentally with test samples containing varied doses of CR3022 and different concentrations of IgG (Fig. 2C); the results obtained were in good agreement with the computational prediction.

We then evaluated the assay with human serum samples: 12 sera from convalescent COVID-19 patients and 2 negative controls from healthy individuals obtained before the pandemic. The samples were serially diluted and analyzed using the tNLuc assay. All convalescent samples produced luminescent signals well above those of controls at all but the highest $(1: 24,300)$ dilutions (Fig. 3A). As was done in Fig. 2C, the overall signal of the samples was calculated as luminescence summation of dilution points 1:300, 1:900 and 1:2700 (Fig. 3B) and showed excellent separation between controls and most convalescent samples.

Finally, we compared the tNLuc assay with several other tests. Similar to the tNLuc assay, ELISA directly measures the antibodies specific to SARS-CoV-2 in blood samples. We performed the ELISA on all 14 samples using a common protocol ${ }^{9}$. The results demonstrated a high degree of correlation with those of the tNLuc assay $\left(R^{2}=0.783\right)$ (Fig. 4A), suggesting that the quantifiability and sensitivity of the tNLuc assay are similar to ELISA. In contrast to direct ELISA, neutralizing antibody assays measure only the antibodies responsible for antagonizing virus-receptor interaction. We therefore carried out two neutralization assays on 10 of the samples: the surrogate virus neutralization test (sVNT) ${ }^{29}$ (Fig. 4B) and the plaque-reduction neutralization test

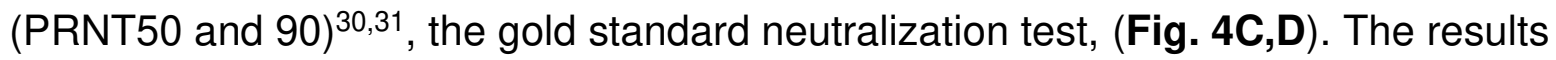


from each of these tests show high positive correlation with the tNLuc assay. Among them, the PRNT90 test produced results highly consistent with those of tNLuC $\left(R^{2}=0.868\right)$. These observations suggest that the tNLuc assay provides a reliable indicator of the neutralization potential of a sample.

\section{Discussion}

In this proof-of-principle study, we generated a novel serological assay for the detection of antibodies against SARS CoV-2. The underlying principle of tNLuc allows the assay to be performed in homogeneous liquid phase, a feature granting rapid detection and exemption from many of the tedious procedures required by ELISA, but without compromising assay quantifiability or sensitivity. In addition, the tNLuc assay can be scaled to high content measurement similar to many CLIAs. In contrast, however, it requires no specialized equipment beyond a regular microplate luminescence reader, making it a highly cost-effective test. Another notable feature is that the tNLuc assay readout shows a high degree of correlation with neutralizing capability. Collectively these features suggest that our assay platform may be of great value for use in clinical laboratories involved in COVID-19 testing although more characterization and evaluation based on larger patient cohort is needed, which is currently being conducted in our lab. Additionally, the tNLuc strategy employed in our assay has the potential to be adapted for use in alternative diagnostic approaches to help assay other pathogens and viruses such as SARS, MERS and/or influenza. 


\section{Methods}

Plasmids and recombinant proteins. To create the general probe, the $\mathrm{C} 2$ domain of protein $\mathrm{G}$ was appended with the $\beta 9$ tag and a $6 x \mathrm{His}$ tag either at its $\mathrm{N}$ - or $\mathrm{C}$-terminus (or both) (Supplementary Fig. 1A). The cDNAs were cloned into pET16b by Gibson assembly. The plasmids were transformed into BL21-Gold (DE3) cells. For expression, the bacteria were grown at $37^{\circ} \mathrm{C}$ until OD550 $0.4-0.6$, followed by further incubation at $22^{\circ} \mathrm{C}$ for 4 hours in the presence of $0.2 \mathrm{mM}$ IPTG. Bacterial cells were collected and lysed by sonication for further purification. Antibody specific probes were created by appending $\beta 10$ tag to $\mathrm{N}$ - or $\mathrm{C}$-, or both termini of SARS-CoV-2 spike protein (ectodomain) or its $\mathrm{RBD}^{32}$. PDGFRB signal peptide was used as $\mathrm{N}$-terminal leading sequence for $\mathrm{N}$-tagged constructs to allow their correct secretion to extracellular space. PolyHis tag was added at their C-termini for purpose of purification. Several modifications were made to the $S$ protein ectodomain probe similar to that previously reported: the foldon sequence was fused to the C-termini to promote trimerization; the furin cleavage site 682RRAR was mutated to GSAS; K986 and R987 were mutated to prolines to stabilize the prefusion conformation (Supplementary Fig. 1B). The plasmids of antibody probes were transfected to HEK 293 cells by PEI and media of cultured cells were collected. Both general probes and antibody probe were purified with $\mathrm{Ni}$ Sepharose (Cytiva 17526801) according to the product manual. CR3022 was produced as described previously ${ }^{33} . \Delta 11 \mathrm{~S}$ was constructed and purified as described ${ }^{20}$.

tNLuc assay. Patient sera or CR3022 were diluted in phosphate buffered saline supplemented with $0.1 \%$ Tween-20 and $0.1 \%$ fatty acid-free bovine serum albumin. In some cases, human IgG (Sigma 14506) was added to mimic human serum sample. The samples were mixed with probes at a final concentration of $150 \mathrm{nM}$ for general probes and $4 \mathrm{nM}$ for antibody probes, and were incubated at room temperature for 30 minutes. An aliquot of the mixture ( $5 \mu \mathrm{l}$ of serum sample or $2 \mu \mathrm{l}$ of CR3022 sample) was mixed with 9 volume of Nano-Glo buffer and substrate (Promega N1110) supplemented with recombinant $\Delta 11 \mathrm{~S}$ (final concentration $10 \mu \mathrm{g} / \mathrm{ml}$ ) in 96 or 384 well microplates. After another 30 minutes of incubation at room temperature, luminescence signals were recorded by a microplate luminometer. CR3022 and probe binding kinetics were mathematically fit with GraphPad Prism 8 using an allosteric sigmoidal model. IgG inhibitory effects on CR3022 binding kinetics was fit using an allosteric non-competitive model:

$$
Y=\frac{Y_{\max } X^{n_{x}}}{X^{n_{x}}\left(1+\frac{I^{n_{i}}}{\alpha K_{i}^{n_{i}}}\right)+K_{m}^{n_{x}}\left(1+\frac{I^{n_{i}}}{K_{i}^{n_{i}}}\right)}
$$


in which $Y$ is the luminescence signal, $X$ is the $C R 3022$ concentration, I is the $\lg G$ concentration, $n_{x}$ is the Hill coefficient for CR3022 and probe interaction, and $n_{i}$ is the Hill coefficient for probe and lgG interaction. The resultant global $R^{2}$ is 0.9911 .

ELISA. ELISA was performed as previously described ${ }^{8,9}$ with some modifications. SARS-CoV-2 spike antigen was immobilized to a 384 well LUMITRAC high binding plate (Greinor Bio-One 781074) by incubating $20 \mu \mathrm{l} /$ well of S- $\beta 10$ protein $(20 \mathrm{nM}$ in PBS) at $37^{\circ} \mathrm{C}$ for 1 hour. After blocking with blocking buffer (PBS supplemented with $0.1 \%$ Tween 20 and $3 \%$ skimmed milk) for one hour at room temperature, the plate was incubated with sera serially diluted in blocking buffer at room temperature for two hours. After three times wash with PBST (PBS supplemented with $0.1 \%$ Tween 20), the plate was further incubated with $\alpha$-human IgG antibody conjugated with horse radish peroxidase (Jackson ImmunoResearch 109-035-098, 1:50,000 in blocking buffer, 30 $\mu \mathrm{l} /$ well) at room temperature for one hour. After three times final wash with PBST, Pico chemiluminescence substrate mixture (Thermo Scientific $3769,30 \mu \mathrm{l} / \mathrm{mL}$ ) was added to the plate and signals were recorded in a microplate luminometer. Data of serially diluted samples were analyzed with GraphPad Prism 8 by model fitting. The total signal was calculated as areas under curve (AUC) of the fitted curves.

sVNT. sVNT kit was purchased from Genscript and the assay was performed according to the manufacturer's instructions ${ }^{29}$. Briefly, diluted serum samples were mixed with HRP-RBD at a $1: 1$ ratio and incubated at $37^{\circ} \mathrm{C}$ for 30 minutes. An aliquot $(100 \mu \mathrm{l})$ of the mixture was moved to a well of a test plate which was precoated with ACE2 provided by the manufacturer, and was incubated at $37^{\circ} \mathrm{C}$ for 15 minutes. After 4 times wash, signal was developed by incubation with TMB solution.

PRNT. PRNT was performed as described previously ${ }^{33}$. Briefly, diluted serum samples were incubated with SARS-CoV-2 virus (50 PFU) in a $\mathrm{CO}_{2}$ incubator for one hour. The mixture was then moved to a well of a 12-well plate cultured with Vero E6 cells (100\% confluency) and incubated for one hour in a $\mathrm{CO}_{2}$ incubator with rocking every 15 minutes. To each well $1.5 \mathrm{ml}$ prewarmed $\left(37^{\circ} \mathrm{C}\right)$ overlay medium (MEM without phenol red but supplemented with 4\% FBS, L-glutamine, nonessential amino acids, sodium bicarbonate and $1.5 \%$ carboxymethycellose) was added followed by incubation for 72 hours. The cells were then fixed with $10 \%$ formalin (neutral-buffered) and subsequently stained with crystal violet $(0.5 \%$ solved in $20 \%$ ethanol). Plaques were counted and compared to negative control. A titre is recorded as the highest serum dilution resulting in $50 \%$ and $90 \%$ reduction in plaques compared with controls. 
Human serum samples. Negative control sera were taken before the pandemic. All patients were diagnosed by SARS-CoV-2 RT-PCR from nasopharyngeal swabs. All samples are de-identified and enrolled through REB approved protocols, REB20-044c or REB 149-1994.

Study approval. The study was approved by the Office of Environmental Health \& Safety at the University of Toronto. All research was performed in accordance with relevant guidelines and regulations. INLuc assay and ELISA were performed at the University of Toronto. sVNT and PRNT were performed at the National Microbiology Laboratory. External samples were transferred through Material Transfer Agreements. All participants have provided informed consent.

Statistics. Statistical analyses were performed using GraphPad Prism 8. The performance difference between $\beta 10-S$ and $\beta 10-S-\beta 10$ was analyzed using an unpaired two-tailed t-test. Correlation between the tNLuc assay and other assays (ELISA, VNT or PRNT) was analyzed using Pearson product-moment correlation. 


\section{References}

1. Tang, Y., Schmitz, J. E., Persing, D. H. \& Stratton, C. W. Laboratory Diagnosis of COVID-19: Current Issues and Challenges. J. Clin. Microbiol. 58, e00512-20 (2020).

2. Peeling, R. W. et al. Serology testing in the COVID-19 pandemic response. Lancet Infect. Dis. 20, e245-e249 (2020).

3. Huang, A. T. et al. A systematic review of antibody mediated immunity to coronaviruses: kinetics, correlates of protection, and association with severity. Nat. Commun. 11, 4704 (2020).

4. Lisboa Bastos, M. et al. Diagnostic accuracy of serological tests for covid-19: Systematic review and meta-analysis. BMJ 370, (2020).

5. Andryukov, B. G. Six decades of lateral flow immunoassay: From determining metabolic markers to diagnosing covid-19. AIMS Microbiol. 6, 280-304 (2020).

6. Whitman, J. D. et al. Evaluation of SARS-CoV-2 serology assays reveals a range of test performance. Nat. Biotechnol. 38, 1174-1183 (2020).

7. Adams, E. R. et al. Antibody testing for COVID-19: A report from the National COVID Scientific Advisory Panel. Wellcome Open Res. 5, 139 (2020).

8. Amanat, F. et al. A serological assay to detect SARS-CoV-2 seroconversion in humans. Nat. Med. 26, 1033-1036 (2020).

9. Stadlbauer, D. et al. SARS-CoV-2 Seroconversion in Humans: A Detailed Protocol for a Serological Assay, Antigen Production, and Test Setup. Curr. Protoc. Microbiol. 57, 1-15 (2020).

10. Bryan, A. et al. Performance Characteristics of the Abbott Architect SARS-CoV-2 IgG Assay and Seroprevalence in Boise, Idaho. J. Clin. Microbiol. 58, 4-11 (2020).

11. Rhoads, D. D. et al. Comparison of abbott id now, diasorin simplexa, and CDC fda emergency use authorization methods for the detection of sars-cov-2 from nasopharyngeal and nasal swabs from individuals diagnosed with covid-19. J. Clin. Microbiol. 58, 17-18 (2020).

12. Package insert of Elecsys Anti-SARS-CoV-2. (2020). doi:10.1093/cid/ciaa344

13. Ainsworth, M. et al. Performance characteristics of five immunoassays for SARSCoV-2: a head-to-head benchmark comparison. Lancet Infect. Dis. 3099, 1-11 (2020).

14. Yao, Z., Petschnigg, J., Ketteler, R. \& Stagljar, I. Application guide for omics approaches to cell signaling. Nat. Chem. Biol. 11, 387-397 (2015).

15. Snider, J. et al. Fundamentals of protein interaction network mapping. Mol. Syst. Biol. 11, 848-848 (2015).

16. Michnick, S. W., Ear, P. H., Manderson, E. N., Remy, I. \& Stefan, E. Universal strategies in research and drug discovery based on protein-fragment complementation assays. Nat. Rev. Drug Discov. 6, 569-582 (2007).

17. Remy, I. \& Michnick, S. W. A highly sensitive protein-protein interaction assay based on Gaussia luciferase. Nat. Methods 3, 977-979 (2006). 
18. Azad, T., Tashakor, A. \& Hosseinkhani, S. Split-luciferase complementary assay: applications, recent developments, and future perspectives. Anal. Bioanal. Chem. 406, 5541-5560 (2014).

19. Ohmuro-Matsuyama, Y. \& Ueda, H. Protein-Protein Interaction Assays Using Split-NanoLuc. in Bioluminescence - Analytical Applications and Basic Biology (IntechOpen, 2019). doi:10.5772/intechopen.86122

20. Dixon, A. S., Kim, S. J., Baumgartner, B. K., Krippner, S. \& Owen, S. C. A Tri-part Protein Complementation System Using Antibody-Small Peptide Fusions Enables Homogeneous Immunoassays. Sci. Rep. 7, 1-13 (2017).

21. Ohmuro-Matsuyama, Y. \& Ueda, H. Homogeneous Noncompetitive Luminescent Immunodetection of Small Molecules by Ternary Protein Fragment Complementation. Anal. Chem. 90, 3001-3004 (2018).

22. Kruger, N. J. Detection of Polypeptides on Immunoblots Using EnzymeConjugated or Radiolabeled Secondary Ligands. in The Protein Protocols Handbook (ed. Walker, J. M.) 405-414 (2002).

23. Zhou, P. et al. A pneumonia outbreak associated with a new coronavirus of probable bat origin. Nature 579, 270-273 (2020).

24. Ju, B. et al. Human neutralizing antibodies elicited by SARS-CoV-2 infection. Nature 584, 115-119 (2020).

25. Yuan, M. et al. A highly conserved cryptic epitope in the receptor binding domains of SARS-CoV-2 and SARS-CoV. Science (80-. ). 368, 630-633 (2020).

26. Cassidy, J. T. \& Nordby, G. L. Human serum immunoglobulin concentrations: Prevalence of immunoglobulin deficiencies. J. Allergy Clin. Immunol. 55, 35-48 (1975).

27. Gonzalez-Quintela, A. et al. Serum levels of immunoglobulins ( $\lg G, \lg A, \lg M)$ in a general adult population and their relationship with alcohol consumption, smoking and common metabolic abnormalities. Clin. Exp. Immunol. 151, 42-50 (2008).

28. Hartman, H., Wang, Y., Schroeder, H. W. \& Cui, X. Absorbance summation: A novel approach for analyzing high-throughput ELISA data in the absence of a standard. PLoS One 13, 1-12 (2018).

29. Tan, C. W. et al. A SARS-CoV-2 surrogate virus neutralization test based on antibody-mediated blockage of ACE2-spike protein-protein interaction. Nat. Biotechnol. 38, 1073-1078 (2020).

30. Mendoza, E. J., Manguiat, K., Wood, H. \& Drebot, M. Two Detailed Plaque Assay Protocols for the Quantification of Infectious SARS-CoV-2. Curr. Protoc. Microbiol. 57, 1-15 (2020).

31. Wang, S., Sakhatskyy, P., Chou, T.-H. W. \& Lu, S. Assays for the assessment of neutralizing antibody activities against Severe Acute Respiratory Syndrome (SARS) associated coronavirus (SCV). J. Immunol. Methods 301, 21-30 (2005).

32. Kim, D. K. et al. A comprehensive, flexible collection of SARS-CoV-2 coding regions. G3 Genes, Genomes, Genet. 10, 3399-3402 (2020).

33. Abe, K. T. et al. A simple protein-based surrogate neutralization assay for SARSCoV-2. JCl insight 5, (2020). 


\section{Acknowledgements}

We thank members in Igor Stagljar's lab for their suggestions. This work was supported by the Toronto COVID-19 Action Fund (Connaught Award \# 0000313897) to Igor Stagljar, and supported by intramural funding from the Office of the Vice President for Research and the $3 i$ Initiative at the University of Utah to Shawn Owen.

\section{Author contributions}

Z.Y. conceptualized the tNLuc assay for antibodies against SARS-CoV-2, was actively involved in most experiments and data analysis, and wrote the bulk of the manuscript. L.D. and F.A. contributed to plasmid preparation and prepared the probes. Z.L. prepared the original cDNA for spike protein and produced CR3022. S.J.K. helped design the assay. H.W., E.J.V. and K.M. performed neutralizing antibody assays. S. P., L.Y., X.L., Z.Z. and F.Y.Y contributed to providing patient samples. J.S. contributed to manuscript writing and assay design. J.T. helped coordinate the project. M.A.D. coordinated the neutralizing antibody assays. A.M., M.O. and S.M. coordinated sample collection and processing. J. R. supervised CR3022 production and contributed to manuscript writing. S.O. is the original inventor of tNLuc system and helped design the tNLuc assay for antibodies against SARS-CoV-2. I.S. guided and supervised the work, contributed to manuscript writing and coordinated the preparation of the manuscript. All authors approved its content.

\section{Competing interests}

I.S. and Z.Y. are named as co-inventors on a patent application concerning the described technology. 


\section{Legend}

Figure 1. tNLuc assay for detecting $\alpha-S A R S-C o V-2$ antibody. A. Schematic workflow of the tNLuc assay. B. Scan of all probe formats/combinations in the tNuc assay using CR3022 Ab (2 $\mu \mathrm{g} / \mathrm{mL}$ ). Results are presented as a heatmap showing RLU values. C-D. CR3022 at different concentrations was tested with the $\beta 9-G$ together with $\beta 10-S(\mathbf{C})$ or $\beta 10-S-\beta 10$ (D) probes. Human IgG, a mouse monoclonal $A b$, and rabbit polyclonal Abs were used as controls. $E$. Comparison of $\beta 10-S$ and $\beta 10-S-\beta 10$ affinities in the tNLuc system. The average Kd values of four experiments for each probe are presented as a bar graph. $\mathrm{P}$ value was calculated using a two tailed t-test.

Figure 2. Inhibitory effect of additional IgG on tNLuc assay. A. Dose response of IgG inhibition. Different amounts of human IgG as indicated were applied to samples containing $2 \mu \mathrm{g} / \mathrm{mL}$ CR3022 followed by analysis with the INLuc assay. B. Inhibition kinetics of IgG in the assay was examined with different doses of CR3022, without IgG or in presence of human $\lg G$ at 25, 50 or $100 \mu \mathrm{g} / \mathrm{mL}$, roughly the amounts in human serum at a 1:200 dilution. C. To mimic serum samples, different amounts of CR3022 $(100,33,11$ or $3.7 \mu \mathrm{g} / \mathrm{mL})$ were spiked into buffer containing background human IgG at concentrations of 5,10 or $20 \mathrm{mg} / \mathrm{mL}$. Each sample was serially diluted and then analyzed with the tNLuc assay. Sums of luminescence readings at 1:300, 1:900 and 1:2700 for each sample are presented.

Figure 3. Detection of $\alpha-S A R S$ CoV-2 antibody in serum samples with the tNLuc assay. Two negative control (NC, grey dashed lines(A) or dots(B)) serum samples and 12 serum samples from convalescent COVID-19 patients were serially diluted as indicated. A. The level of antiSARS CoV-2 antibodies at each dilution was measured using the tNLuc test. B. The overall signal of antibodies in each sample was calculated by summation of luminescent signals at dilutions 1:300, 1:900 and 1:2700.

Figure 4. Comparison of tNLuc assay with ELISA and neutralizing antibody assays. A. The same samples in Fig. 3 were tested using ELISA and the results are compared with tNLuc using scatter plot. B-D. Ten sera were subject to neutralizing antibody tests: sVNT (B), PRNT50 (C) and PRNT90 (D). $\mathrm{R}^{2}$ and P-value were obtained from Pearson correlation analysis. 
Fig. 1

A

Step 1.

Incubate with probes for $30 \mathrm{~min}$

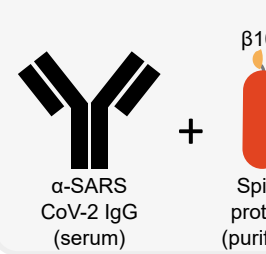

B

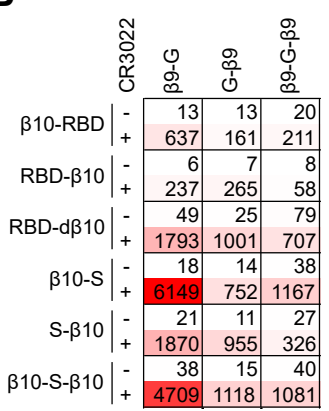

c

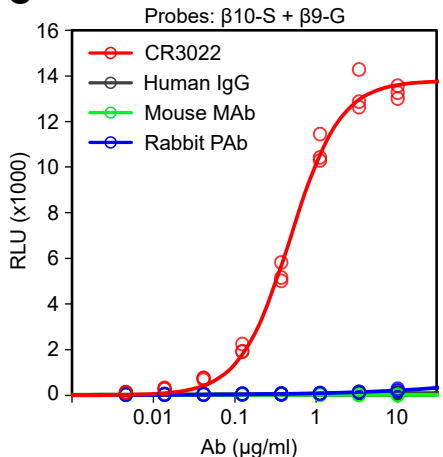

Step 2.

Incubate with $\Delta 11 \mathrm{~S}$ and substrate for $30 \mathrm{~min}$.
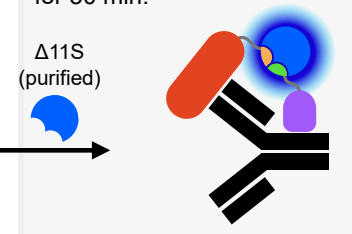

D

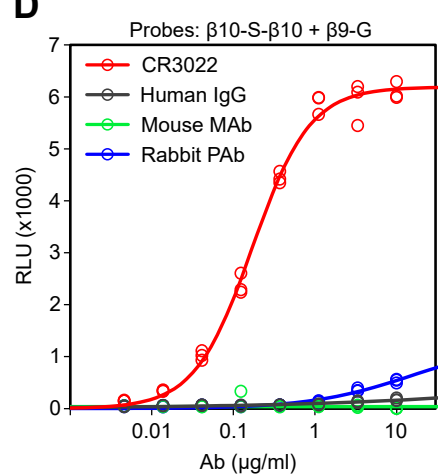

E

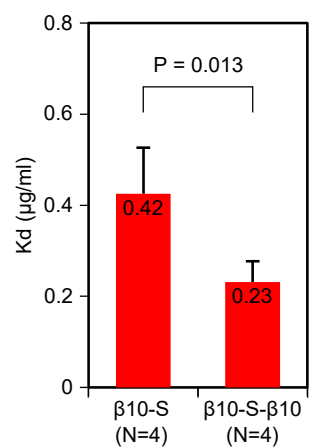


Fig. 2
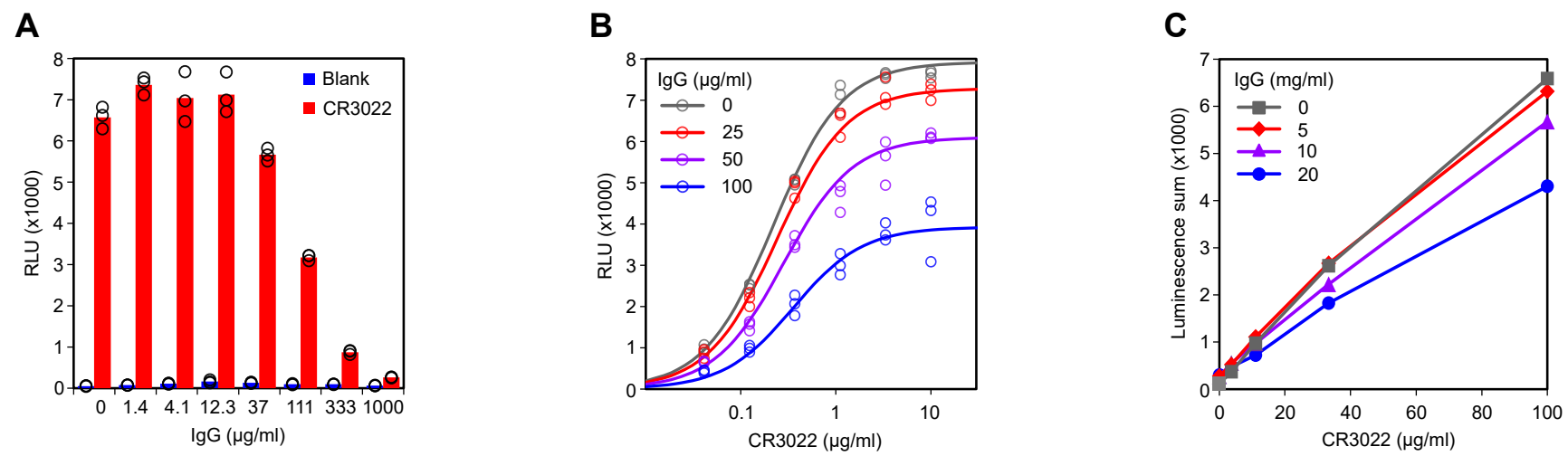
Fig. 3

A

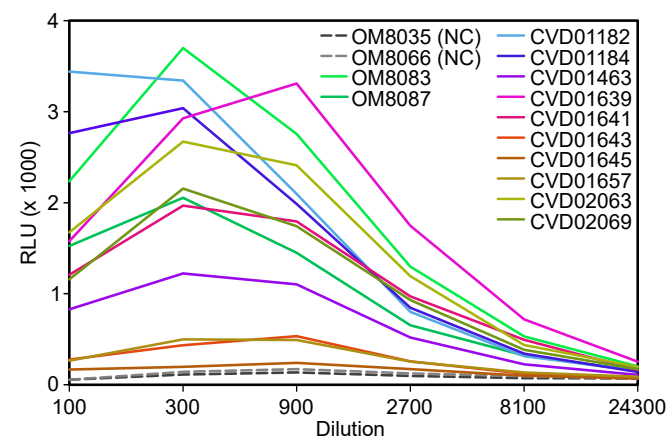

B

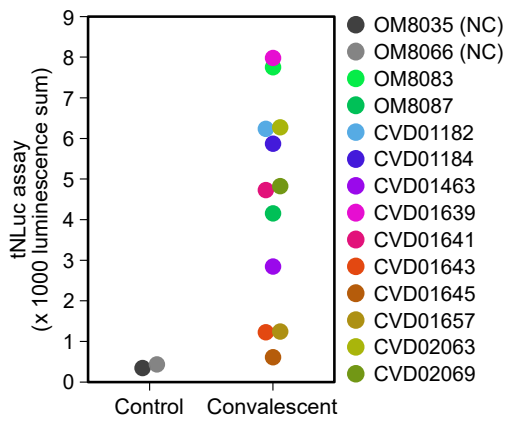


Fig. 4

A

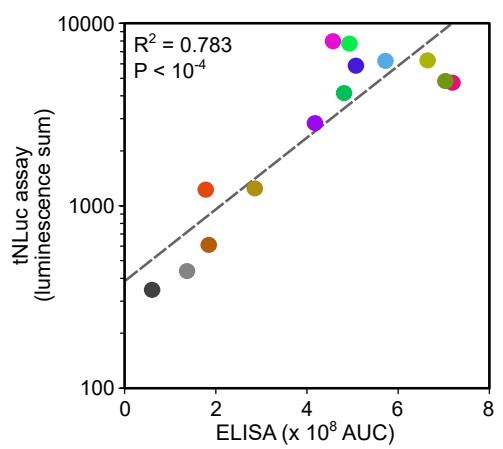

OM8035 (NC)

OM8066 (NC)

OM8083

OM8087

CVD01182

- CVD01184

- CVD01463

CVD01639

- CVD01641

CVD01643

CVD01645

CVD01657

CVD02063

- CVD02069

B

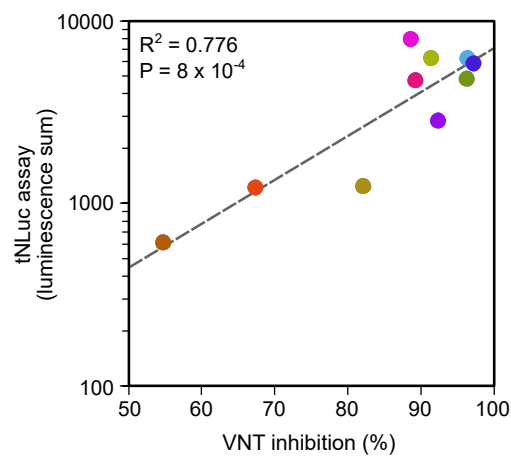

CVD01182

- CVD01184

CVD01463

CVD01639

CVD01641

CVD01643

CVD01645

- CVD01657

CVD02063

CVD02069

C

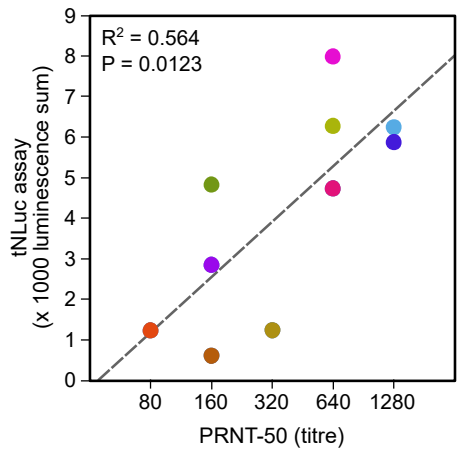

D

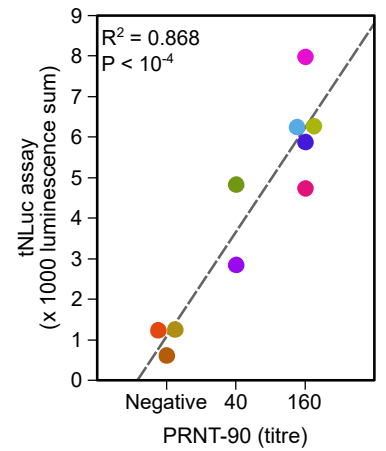




\section{Supplementary Fig. 1}

$\beta 9-G$

B9 Protein $\mathrm{G}$ (C2 domain) $\quad$ 6xHis

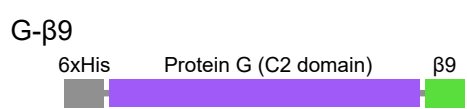

$\beta 9-G-\beta 9$
$\beta 9$$\quad$ Protein $\mathrm{G}(\mathrm{C} 2$ domain) $\quad \beta 96 \times$ His

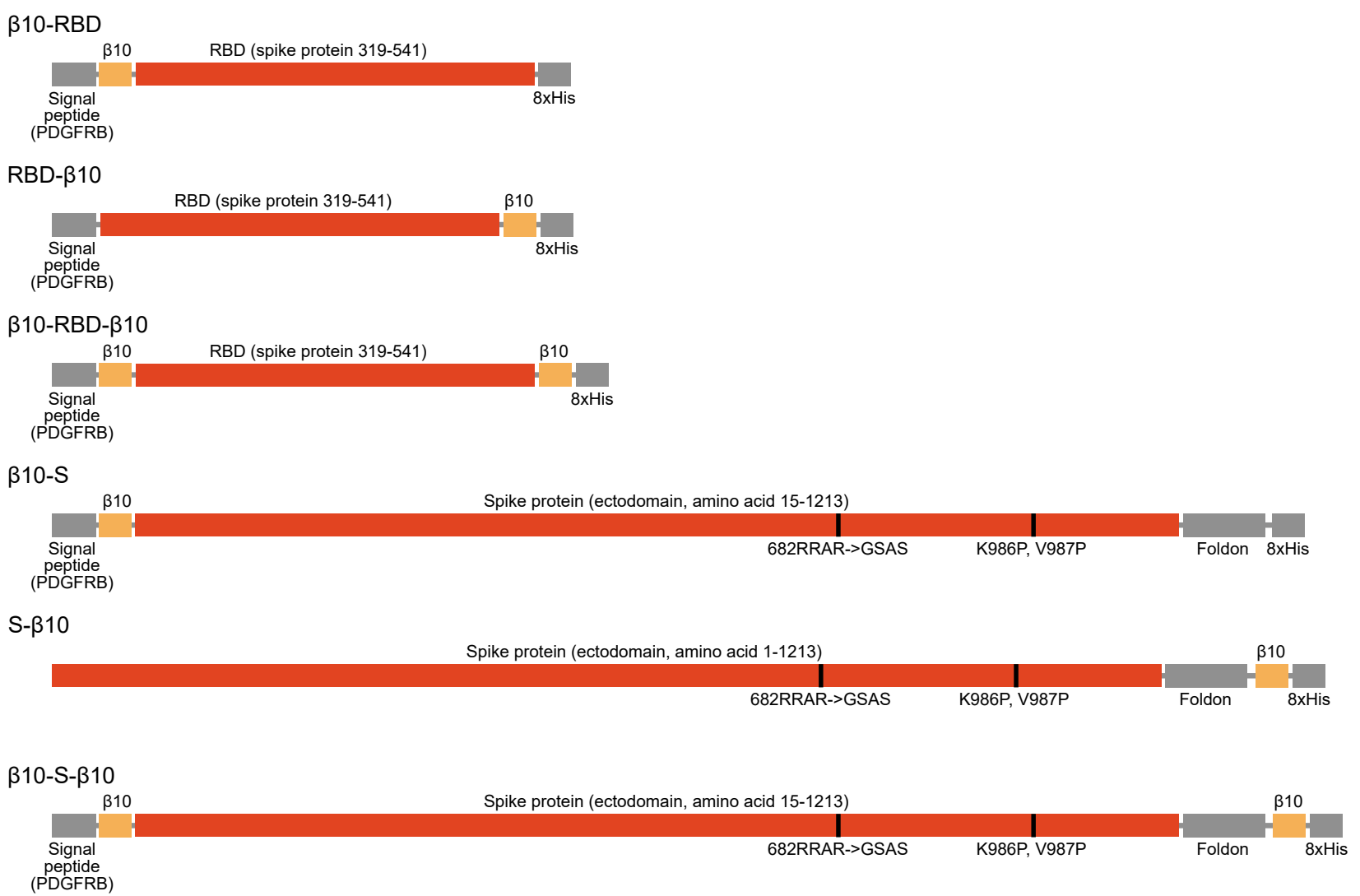

Supplementary Figure 1. Schematic of tNLuc probes. 


\section{Supplementary Fig. 2}

A
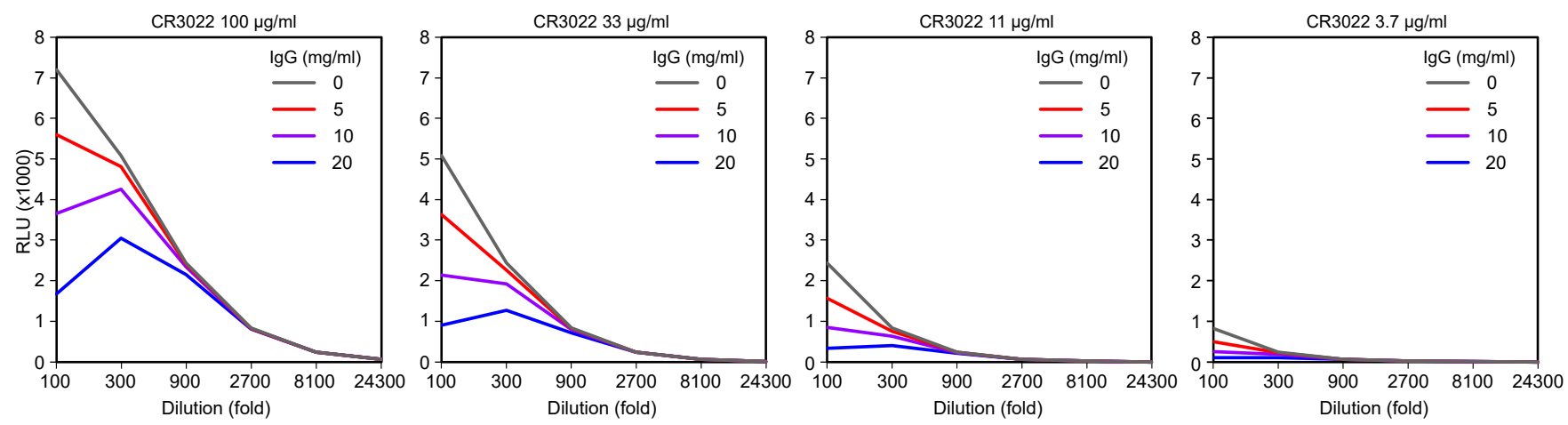

B

C
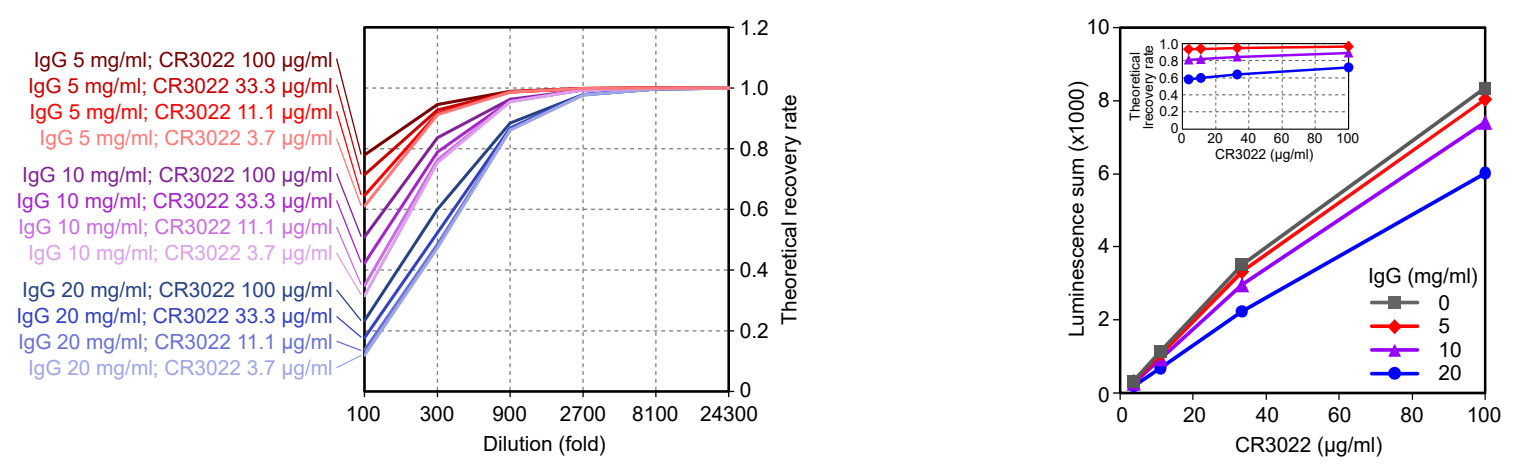

Supplementary Figure 2. Computational simulation of inhibitory effects of IgG on the tNLuc assay. A. Virtual samples containing CR3022 $(3.7,11,33$ and $100 \mu \mathrm{g} / \mathrm{mL})$ in the presence of $\lg$ at different levels $(5,10$, and $20 \mathrm{mg} / \mathrm{mL})$ were serially diluted as indicated. Their tNLuc signals at each dilution endpoint were calculated using the chemical kinetics model derived from the experimental data shown in Fig. 2B. B. Theoretical recovery rates of samples in $\mathbf{A}$ at different dilutions were calculated by dividing the calculated reading of a virtual sample in $\mathbf{A}$ by the corresponding virtual sample not containing IgG. C. The overall tNLuc signal of each virtual sample was calculated by luminescence summation of the calculated signals at the 1:300, 1:900 and 1:2700 dilutions obtained in $\mathbf{A}$. Overall recovery rates are presented inset. 
A

Step 1.

Incubate with probes for $30 \mathrm{~min}$.

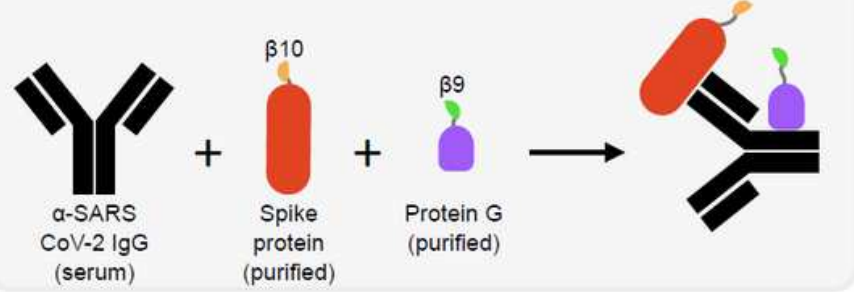

B

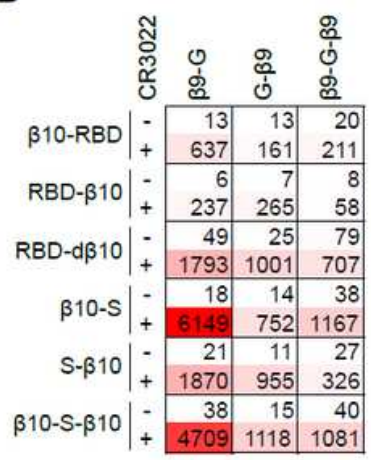

C

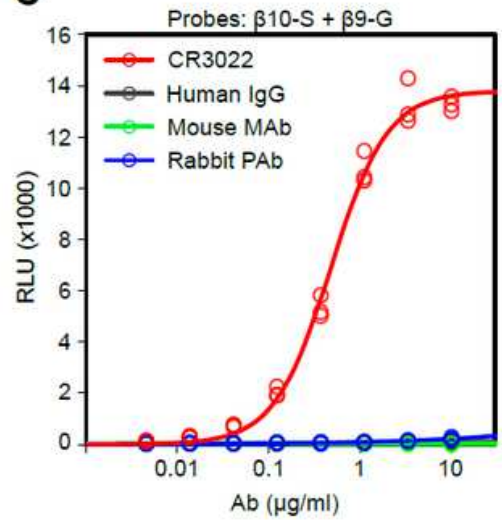

Step 2.

Incubate with $\Delta 11 S$ and substrate for $30 \mathrm{~min}$.

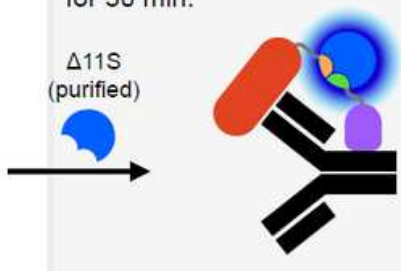

Reading

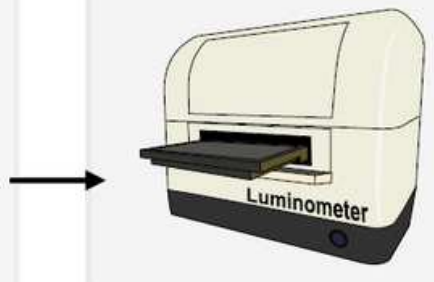

D

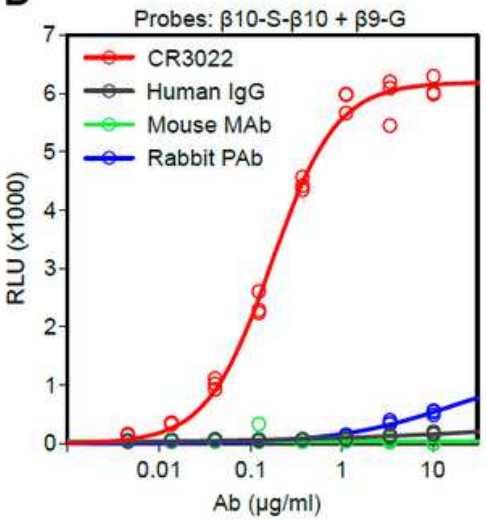

E

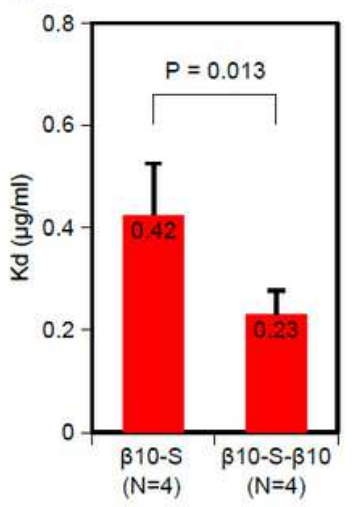

Figure 1

tNLuc assay for detecting a-SARS-CoV-2 antibody. A. Schematic workflow of the tNLuc assay. B. Scan of all probe formats/combinations in the tNuc assay using CR3022 $\mathrm{Ab}(2 \mu \mathrm{g} / \mathrm{mL})$. Results are presented as a heatmap showing RLU values. C-D. CR3022 at different concentrations was tested with the $\beta 9-G$ together with $\beta 10-S$ (C) or $\beta 10-S-\beta 10$ (D) probes. Human IgG, a mouse monoclonal Ab, and rabbit polyclonal Abs were used as controls. E. Comparison of $\beta 10-S$ and $\beta 10-S-\beta 10$ affinities in the tNLuc system. The average $\mathrm{Kd}$ values of four experiments for each probe are presented as a bar graph. $\mathrm{P}$ value was calculated using a two tailed t-test.

A

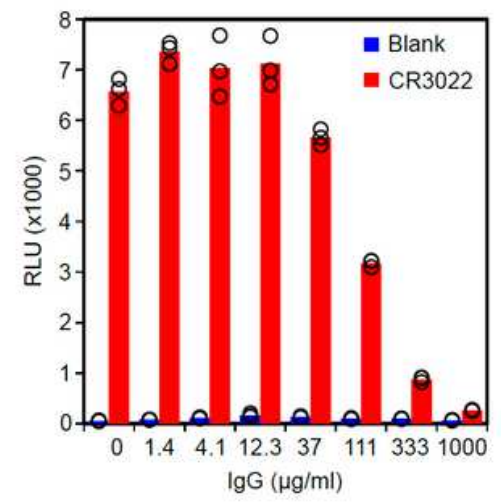

B

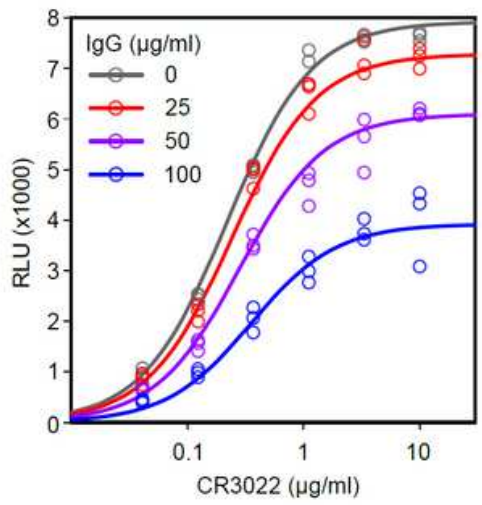

C

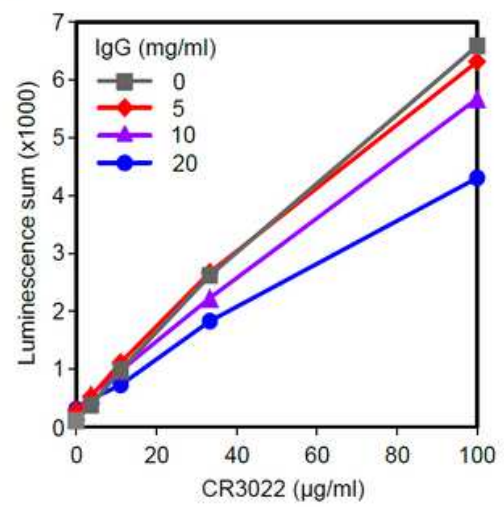




\section{Figure 2}

Inhibitory effect of additional IgG on tNLuc assay. A. Dose response of IgG inhibition. Different amounts of human IgG as indicated were applied to samples containing $2 \mu \mathrm{g} / \mathrm{mL}$ CR3022 followed by analysis with the tNLuc assay. B. Inhibition kinetics of IgG in the assay was examined with different doses of CR3022, without IgG or in presence of human IgG at 25,50 or $100 \mu \mathrm{g} / \mathrm{mL}$, roughly the amounts in human serum at a 1:200 dilution. C. To mimic serum samples, different amounts of CR3022 (100, 33, 11 or 3.7 $\mu \mathrm{g} / \mathrm{mL}$ ) were spiked into buffer containing background human $\lg G$ at concentrations of 5,10 or 20 $\mathrm{mg} / \mathrm{mL}$. Each sample was serially diluted and then analyzed with the tNLuc assay. Sums of luminescence readings at 1:300, 1:900 and 1:2700 for each sample are presented.

\section{A}

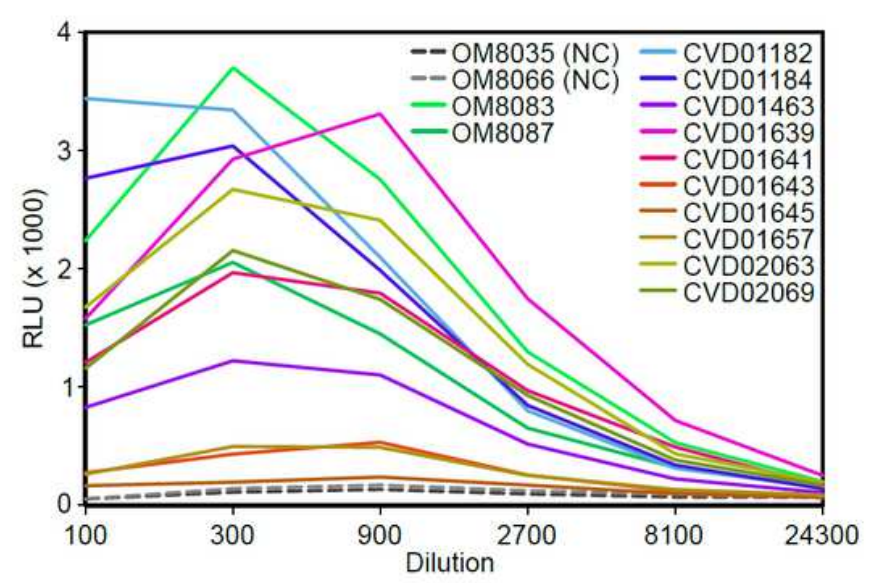

B

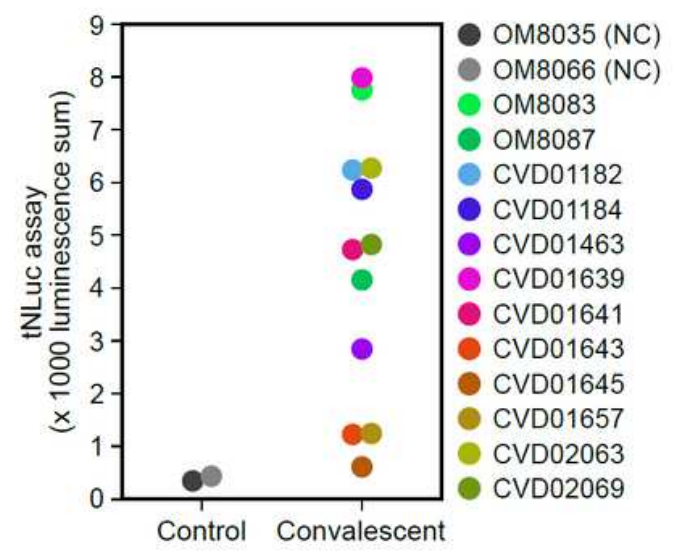

\section{Figure 3}

Detection of a-SARS CoV-2 antibody in serum samples with the tNLuc assay. Two negative control (NC, grey dashed lines(A) or dots(B)) serum samples and 12 serum samples from convalescent COVID-19 patients were serially diluted as indicated. A. The level of anti-SARS CoV-2 antibodies at each dilution was measured using the tNLuc test. B. The overall signal of antibodies in each sample was calculated by summation of luminescent signals at dilutions 1:300, 1:900 and 1:2700. 
A

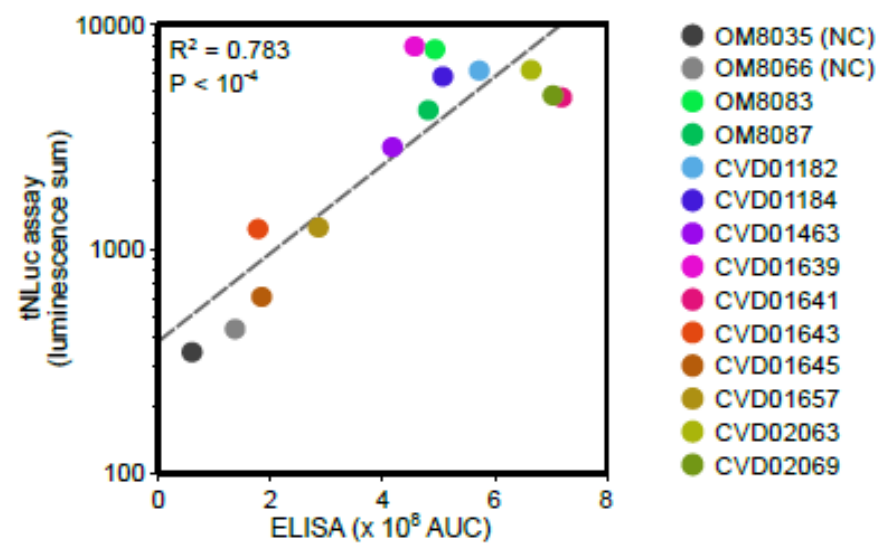

B

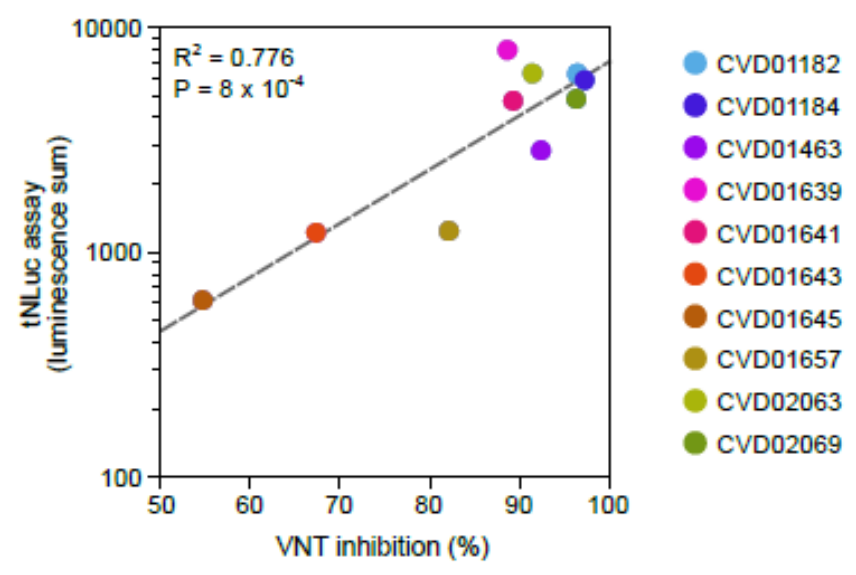

C

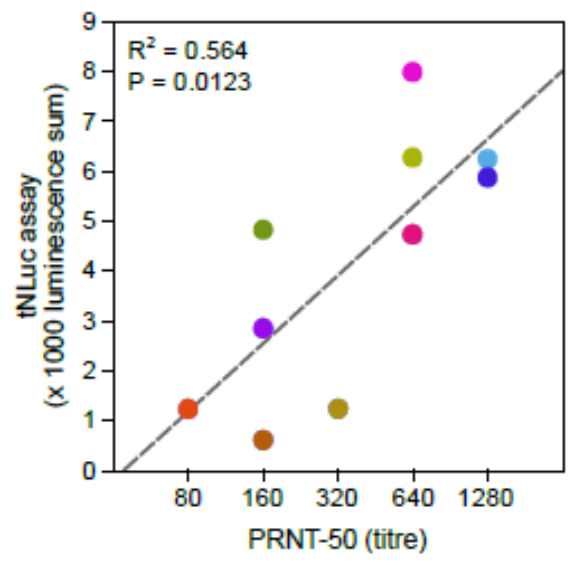

D

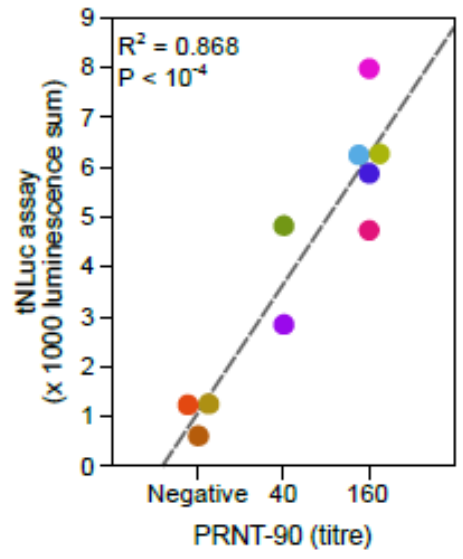

\section{Figure 4}

Comparison of tNLuc assay with ELISA and neutralizing antibody assays. A. The same samples in Fig. 3 were tested using ELISA and the results are compared with tNLuc using scatter plot. B-D. Ten sera were subject to neutralizing antibody tests: sVNT (B), PRNT50 (C) and PRNT90 (D). R2 and P-value were obtained from Pearson correlation analysis. 